\title{
Review: intra-articular corticosteroid injections are better than placebo for improving symptoms of knee osteoarthritis
}

Arroll B, Goodyear-Smith F. Corticosteroid injections for osteoarthritis of the knee: meta-analysis. BMJ 2004;328:869.

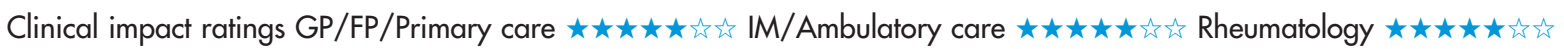
In patients with osteoarthritis (OA) of the knee, are intra-articular corticosteroid injections more effective than placebo
for improving symptoms?

\section{METHODS}

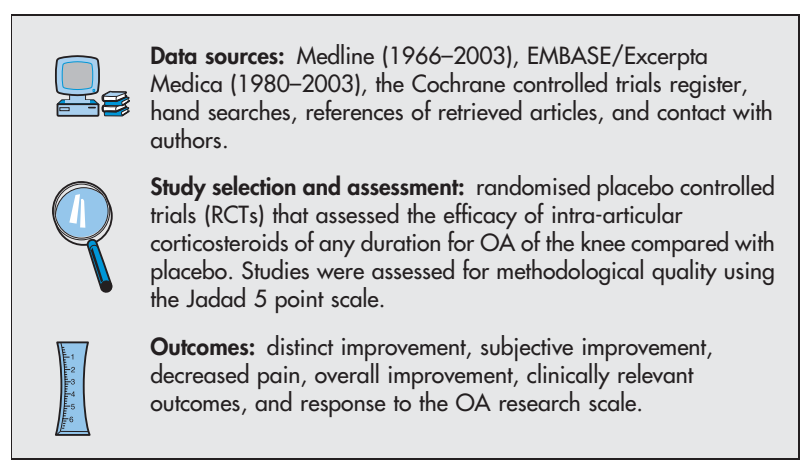

\section{MAIN RESULTS}

10 studies met the selection criteria $(\mathrm{n}=546)$. The corticosteroids studied were meticortelone, triamcinolone hexacetonide, methylprednisolone, hydrocortisone, and cortivazol. The meta-analysis of 6 pooled studies showed that patients who received intra-articular corticosteroid injections had greater short term (up to 2 weeks) improvement of symptoms than did those who received placebo (table). The pooled results of 2 high quality studies (Jadad score $=$ 5) also showed long term (16-24 weeks) improvement in OA symptoms (table). However, the individual results of these 2 studies did not differ for OA symptom outcomes. 5 studies $(n=283)$ that used a visual analogue scale showed improvement in pain up to 2 weeks after corticosteroid injections compared with placebo (weighted mean difference $-16.47,95 \%$ CI -22.92 to -10.03 ).

For correspondence: Dr B Arroll, University of Auckland, Auckland, $\ddot{\text { New }}$ Zealand. b.arroll@auckland.ac.nz

Source of funding: New Zealand Accident Rehabilitation and Compensation Insurance Corporation.

\section{CONCLUSION}

In patients with osteoarthritis of the knee, intra-articular corticosteroid injections are more effective than placebo for improving symptoms in the short and long term.

\section{Commentory}

Tre he biological mechanism of corticosteroids suggests that they should be effective in joint disease with significant inflammation-a fact established in the treatment of rheumatoid arthritis. However, how beneficial are corticosteroids in such conditions as OA? The metaanalysis by Arroll et al provides the best answers to date: (1) Intraarticular steroids are modestly better than saline for short term relief of pain. Patients who receive intra-articular corticosteroids are 1.6 times more likely to improve at 2 weeks than those who receive placebo injections (number needed to treat [NNT] =4). This conclusion is robust, given the results of previous studies, ${ }^{1}$ the tendency of OA to worsen over time, and the consistency of the findings of this review $(7$ of 10 trials showed improvement). (2) Corticosteroids appear to have slight benefit at 16-24 weeks (NNT =5). However, this conclusion was based on only 2 trials, is less consistent with the clinical experience of rheumatologists, and may be dose dependent.

The review by Arroll et al also raises several questions: which group of OA patients are likely to respond to corticosteroids (ie, those with less severe disease or those with clinical evidence of inflammation such as an effusion)? To what degree is the apparent success of intra-articular steroids affected by how the procedure is performed? For example, how much fluid is withdrawn if lavage is used rather than saline instillation? At what point in the treatment regimen should intra-articular corticosteroids be used (ie, after or before NSAID or physical therapy)? What is the effective and safe interval for repeat injections?

Unless or until further studies address these issues, clinicians should be reassured that with prudent use, intra-articular corticosteroids remain a valuable option for short term management of OA of the knee.

Stanford Shoor, MD Kaiser Permanente Medical Center Santa Clara, California, USA

1 Wise C. The rational use of steroid injections in arthritis and nonarticular musculoskeletal pain syndromes. Bull Rheum Dis 2003;52:1-4.

Intra-articular corticosteroid injections (ICI) v placebo for osteoarthritis of the knee*

\begin{tabular}{llllll}
\hline & & \multicolumn{2}{l}{ Weighted event rates } & & \\
\cline { 3 - 4 } Outcomes & Number of studies $(\mathbf{n})$ & ICI & Placebo & RBI (95\% CI) & NNT (CI) \\
\hline Short term improvement & $6(317)$ & $78 \%$ & $45 \%$ & $53 \%$ (21 to 93) & 4 (3 to 6) \\
Long term improvement & $2(97)$ & $45 \%$ & $21 \%$ & $110 \%$ (20 to 266) & 5 (3 to 14) \\
\hline
\end{tabular}

*Short term improvement $=$ up to 2 weeks; long term improvement $=16-24$ weeks. Other abbreviations defined in glossary; RBI, NNT, and Cl calculated from data in article using a random effects model. 